\title{
Genetics of Root System Architecture Using Near-isogenic Lines of Melon
}

\author{
Ana Fita ${ }^{1}$ and Belén Picó \\ Institute for the Conservation and Breeding of Agricultural Biodiversity (COMAV), Universidad \\ Politécnica de Valencia, Camino de Vera s/n, 46022, Valencia, Spain \\ Antonio J. Monforte \\ IRTA, Centre de Recerca en Agrigenòmica, Ctra. de Cabrils, E-08348 Cabrils (Barcelona), Spain
}

Fernando Nuez

Institute for the Conservation and Breeding of Agricultural Biodiversity (COMAV), Universidad

Politécnica de Valencia, Camino de Vera s/n, 46022, Valencia, Spain

\begin{abstract}
AdDitional INDEX words. allometry, Cucumis melo, root morphology, soil stress resistance, ssp. agrestis
ABstract. The study of the genetic control of natural variation in the root architecture of Cucumis melo $\mathrm{L}$. is complex due to the difficulties of root phenotyping and to the quantitative nature of root traits and their plasticity. A library of near-isogenic lines (NILs), constructed by introgressing the genome of the exotic Korean accession Shongwan Charmi [SC (PI161375)] into the genetic background of the cultivar Piel de Sapo (PS) has recently become available. In this work, we used this population to identify quantitative trait loci (QTLs) controlling variation in root growth and architecture. We studied separately the primary root and the secondary and tertiary root systems during a 15-day period. Heritabilities for the root traits were moderate. Correlation and principal component analysis showed independence among traits measuring root length and root branching level, indicating the possibility of modifying both traits independently. PS and SC clearly differed in plant size. Significant allometric relationships between vine biomass and some root traits were identified. The use of NILs with similar plant size of PS allowed us to avoid the inaccuracies caused by size-dependent variation of root traits. A total of 17 QTLs for root traits in seven linkage groups were identified: three QTLs for primary root length, three QTLs for the diameter of the primary root, three QTLs for secondary root density, three QTLs for the average length of the secondary roots, three QTLs for the percentage of secondary roots bearing tertiary roots, and two QTLs for tertiary root density. In most of these traits, transgressive variation was observed.
\end{abstract}

The root system plays a very important role in plant development. A root system that is strong, healthy, and has an adequate architecture for the environment in which it develops provides numerous benefits to the plant, such as a greater capacity to absorb water and nutrients, greater exposure to beneficial soil microorganisms, and a higher tolerance to biotic or abiotic soil stress (Clarke and McCaig, 1993; Fita et al., 2007; Fitter, 2002; Lynch, 1995).

The breeding of crops for more efficient root systems has traditionally been neglected due to the difficulties of phenotyping roots and of linking root form and function to crop productivity. To breed root systems, it is necessary to have genetic variation available for the favorable traits as well as reliable tools to determine the genetic control of this variation. The quantitative nature of root traits, their complex genetic control, and the strong environmental effects on those traits

Received for publication 17 Dec. 2007. Accepted for publication 20 Mar. 2008. We thank Eva Martinez (COMAV) and Cristina Roig for their technical assistance.

This work was supported by MCT AGL2003-04817 and GEN2003-20237C06-03.

A.F. carried out the in vitro root analysis, B.P. conceived, designed, and coordinated the study, A.F. and B.P. carried out data analysis and drafted the manuscript, A.J.M. developed the NIL collection, providing it for this study, and critically reviewed the manuscript, F.N. is the director of COMAV and reviewed the manuscript, and all authors read and approved the final manuscript.

${ }^{1}$ Corresponding author. E-mail: anfifer@btc.upv.es. make traditional genetic studies difficult (Harper et al., 1991; Malamy, 2005).

Mutant screenings have provided information about some genes involved in root development (reviewed in Picó et al., 2007). Additionally, several research groups have analyzed the existing natural variation by mapping quantitative trait loci (QTLs) that control different aspects of root system architecture and development. These studies mostly have been carried out in the model species Arabidopsis thaliana (L.) Heynh (FitzGerald et al., 2006; Loudet et al., 2005), although rice (Oryza sativa L.; Li et al., 2005; Steele et al., 2006; Zheng et al., 2003), maize (Zea mays L.; Landi et al., 2007; Tuberosa et al., 2003; Zhu et al., 2005), bean (Phaseolus vulgaris L.; Beebe et al., 2006; Ochoa et al., 2006), and lettuce (Lactuca sativa L.; Argyris et al., 2005; Johnson et al., 2000), and some other crops have also been studied (reviewed in Picó et al., 2007). This has been possible through the availability of appropriate populations, such as recombinant inbred lines, advanced backcross populations (Tanksley and Nelson, 1996), and genomic libraries of near-isogenic lines (NILs; Eshed and Zamir, 1995), which allow for an accurate cartography of quantitative traits (Tuberosa et al., 2003).

Soil stresses are responsible for worldwide economic losses in melon cultivation that run into millions of dollars (GómezGuillamón and Álvarez, 2001). Unfortunately, root morphology studies on melon (Cucumis melo) to diminish those detrimental effects have not been conducted systematically, probably due to the lack of appropriate tools. In previous assays 
(Dias et al., 2004; Fita et al., 2006; A. Fita, B. Picó, and F. Nuez, unpublished), we have studied the root architecture variation in the two subspecies into which C. melo is divided: ssp. melo L. and ssp. agrestis (Naudin) Pangalo (Jeffrey, 1980). Most commercial cultivars that are popular in Europe and the U.S. belong to the inodorus group (C. melo ssp. melo var. inodorus H. Jacq.) and the cantalupensis group (C. melo ssp. melo var. cantalupensis Naudin) of ssp. melo, whereas the ssp. agrestis includes exotic, weedy, and wild germplasm. Wild relatives of the cultivated plants have frequently been used as a source of genes for favorable root traits, as they are adapted to stressful conditions. We have found extensive variation between ssp. melo and ssp. agrestis for primary root growth and secondary root system in different environments. The genetic basis of these differences has been studied using an F2:3 population derived from the cross 'Piel de Sapo' (PS; C. melo ssp. melo var. inodorus) $\times$ Pat 81 [C. melo ssp. agrestis var. conomon (Thunb.) Makino] (Dias et al., 2004; Fita et al., 2006).

A genomic library of NILs of melon was recently developed by introgressing single-chromosome regions from the exotic Korean accession 'Shongwan Charmi' (SC; PI161375), which belongs to ssp. agrestis var. conomom, into the genetic background of PS (Eduardo et al., 2005). These NILs have the additional advantage over recombinant inbred lines of allowing the study of the effects of the different introgressions of the var. conomon genome in a uniform var. inodorus PS genetic background. This population has already been successfully used to study the genetics of fruit quality and postharvest physiological fruit disorders, even allowing the identification of QTL alleles with beneficial effects masked in the var. conomon background (Eduardo et al., 2007; Fernández-Trujillo et al., 2007; Obando et al., 2008). This NIL library is especially advantageous in studying the genetics of root traits in these two taxa, as conomon and inodorus types not only differ in root architecture, but also exhibit different growth patterns and plant size. Comparing different-sized plants can be difficult, and allometric relationships are usually used to better differentiate real variation in root parameters from variation due only to differences in size (Johnson et al., 2000; Ochoa et al., 2006; Zhu and Lynch, 2004). The use of segregant populations with similar genetic backgrounds will likely facilitate the analysis.

We studied the genetics of natural variation in root architecture found in C. melo using NILs, analyzing traits considered to have a significant effect on soil exploratory efficiency. A total of 17 QTLs were detected, representing intrinsic developmental variation of root architecture in this material under standard in vitro growth conditions. This is, to the best of our knowledge, the first study on the genetics of root architecture in C. melo using NILs.

\section{Materials and Methods}

Plant material. The plant material used in this study was a set of 26 NILs derived from a cross between two melon cultivars: a Spanish cultivar, Piel de Sapo (C. melo ssp. melo var. inodorus), and the exotic Korean accession Shongwan Charmi (PI161375; C. melo ssp. agrestis var. conomon). These NILs were developed by marker-assisted selection in a previous work (Eduardo et al., 2005). The NILs had marked defined introgressions from SC into the PS background, which covered most of the SC genome (Eduardo et al., 2007) and were coded with the prefix "SC." The first number indicates the linkage group (LG), and the second one indicates the number of the NIL within the LG. Further details about the number of backcrosses and the density of the map used to construct these NILs are given in Eduardo et al. (2005).

EXPERIMENTAL DESIGN AND PHENOTYPIC EVAluation. A nondestructive assay was used to monitor root development using vertical agar in vitro plates. Fifteen seeds of each NIL (and 20 of the SC and PS) were sterilized using 50\% bleach and Tween-20 (Panreac, Barcelona, Spain) for $20 \mathrm{~min}$, and were then washed with sterile water three times. Subsequently, seeds were sown in tubes with Murashige-Skoog salts including vitamins (Duchefa Biochemie, Haarlem, The Netherlands), with $30 \mathrm{~g} \cdot \mathrm{L}^{-1}$ of sucrose, $8 \mathrm{~g} \cdot \mathrm{L}^{-1}$ of agar (Pronadisa, Madrid, Spain), and $200 \mathrm{mg} \cdot \mathrm{L}^{-1}$ of cefotaxime (Duchefa Biochemie) to avoid endogenous bacterial contaminations (C. Roig, personal communication). After $48 \mathrm{~h}$ in the dark (day 0), the germinated seeds were transferred to $23 \times 19-\mathrm{cm}$ rectangular plates containing $300 \mathrm{~mL}$ of the same solid medium as the tubes, without cefotaxime. The plates were sealed with cotton to ensure air renewal. The plates were placed vertically on racks in a growth room (16/8 h, light/dark) at a constant temperature of $25{ }^{\circ} \mathrm{C}$. Plates with plants of each genotype were randomly distributed in the growth room. High-resolution digital images (400 dots per inch) of each plant root were taken over a 15 -d period (days 1, 4, 6, 8, 11, and 15 after transplanting) using a scanner (model LA 1600+; Epson America, Long Beach, CA) using transmitted light mode. Plants from seeds that did not germinate at day 0 as well as those with a root system which stayed on the media surface were not considered.

Traits MEASURED. The weight of each seed was measured before starting the assay [SW (in milligrams)]. At the end of the assay, the vine of each plant was cut and weighed [VW (in grams)]. The analyzed root traits were classified into four categories (Zobel, 2005a, 2005b): 1) whole root system (WRS); 2) primary root (PR); 3) secondary root system (SRS; lateral roots emerging from the PR); and 4) tertiary root system (TRS; lateral roots emerging from secondary roots). The images of the roots were edited manually to accurately analyze each trait within each root category (Fig. 1).

Within each root category, root traits were evaluated using the specific software for root analysis WinRhizo Pro 2003b (Régent Instruments, Québec, Canada). The number of roots of different classes was counted manually. For the WRS, the following parameters were evaluated $(1,4,6,8,11$, and $15 \mathrm{~d})$ : total root length [RL (in centimeters)], total projected area [PA (in squared centimeters)], average diameter [AD (in millimeters)], and total number of tips (TT) as an indirect measurement of the branching degree of the root. For the PR, the following parameters were evaluated $(1,4,6$, and $8 \mathrm{~d})$ : PR length [PRL (in centimeters)] and average diameter of PR [PAD (in millimeters)]. For the SRS, the following parameters were evaluated $(4,6$, and $8 \mathrm{~d})$ : total length of the SRS, as the sum of the length of all of the lateral roots belonging to the SRS [SRL (in centimeters)]; average diameter of the SRS [SAD (in millimeters)]; secondary root number, as the number of each discernible lateral secondary root $>1 \mathrm{~mm}$ emerging from the PR (SRN); secondary root density, calculated as SRN/PRL [SRDe (SRN per centimeter)]; and the average length of 

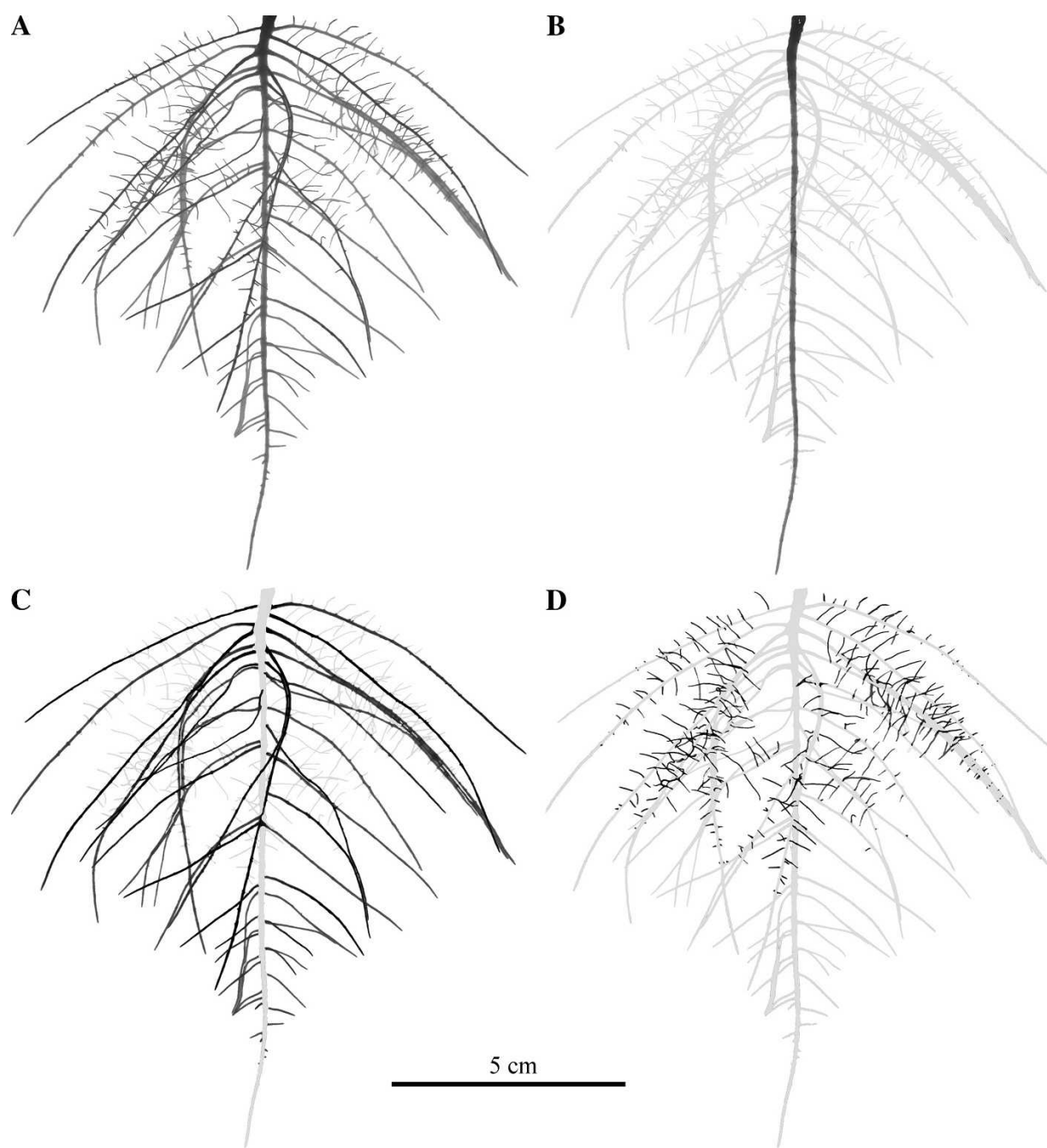

Fig. 1. Cucumis melo 'Piel de Sapo' root $8 \mathrm{~d}$ after transplanting, showing the different root classes analyzed: (A) whole root system, $(\mathbf{B})$ primary root, $(\mathbf{C})$ secondary root system, and (D) tertiary root system.
Phenotypic correlations between all root traits were calculated as Pearson correlation coefficients $\left(r_{p}\right)$ between traits independently in each date. Genotypic correlations $\left(r_{g}\right)$ were also estimated as

$$
r_{g}=\frac{\sigma_{b(x, y)}}{\sigma_{b(x)} \sigma_{b(y)}}
$$

where $\sigma_{b}(x, y)$ is the covariance between NILs for traits $x$ and $y$, and $\sigma_{b}(x)$ and $\sigma_{b}(y)$ are the standard deviations for the variance between NILs for traits $x$ and $y$. The statistical significance of the genotypic correlations was estimated using the method proposed by Hébert et al., (1994).

To characterize the size-dependent variation of root traits, the allometric relationships between root traits and VW were studied. Allometric relationships determine how different parameters of an organism scale with one another. Adapting the formula of Hunt (1990), our formula for calculating allometric relationships among root and vine traits is

$$
\log \mathrm{Y}=\log \mathrm{b}+\mathrm{K} \log \mathrm{X}
$$

where $\mathrm{Y}$ is the root trait, $\mathrm{b}$ is $\mathrm{a}$ normalization constant, $\mathrm{K}$ is the allometric coefficient, and $\mathrm{X}$ is the VW. These relationships were determined from paired measurements of root traits and VW by linear regression in the NILs population. The value of the coefficient of determination $\left(r^{2}\right)$ secondary roots, calculated as the ratio SRL/SRN [ALSR (in centimeters)]. For the TRS, the following parameters were evaluated $(4,6$, and $8 \mathrm{~d})$ : number of secondary roots bearing tertiary roots (SRT); the proportion of laterals bearing tertiaries, calculated as the ratio SRT/SRN (\%SRT); the number of tertiary roots (TRN); and the density of tertiary roots calculated as the ratio TRN/SRT (TRDe). All of these traits were measured using the digital images of a minimum of 10 plants per line.

Data analysis. Statistical analyses were performed with SPSS (version 14.0 for Windows; SPSS Inc., Chicago) and Excel 2002 (Microsoft Corp., Redmond, WA). Heritability $\left(h^{2}\right)$ was estimated for each trait and date independently as

$$
h^{2}=\sigma_{\mathrm{b}}^{2} / \sigma_{t}^{2}
$$

where $\sigma^{2}{ }_{b}$ and $\sigma^{2}$ are the between-line (which estimates the genotypic variance) and total variances (which estimates the phenotypic variance), respectively, estimated from the mean squares of the analysis of variance. The estimates of the standard error were calculated according to Kempthorne's method (Kempthorne, 1969). indicates if the best scaling relationship accounts for a significant amount of variation in the root trait. Low $r^{2}$ suggests that the root trait does not scale with VW.

A principal component analysis (PCA) was also conducted using NTSYSpc software (version 2.02g; Applied Biostatistics, Setauket, NY) to determine if a combination of attributes could better explain phenotypic diversity (Langlade et al., 2005). The first three PCA axes were studied. The first principal component (PC1) represents the greatest amount of variation resulting from a combination of the variables. The second principal component (PC2) represents the next greatest source of variation, and so on. Traits with eigenvectors $>0.50$ were considered the most significant contributors to each PC.

To study the effect of each SC introgression, NIL mean values for each day were compared with the control genotype PS using a Dunnet's contrast (Dunnet, 1955) with type-I error $\alpha \leq 0.05$. The number of QTLs was estimated as in Eduardo et al. (2007), assuming that there was, at least, one QTL in the introgression of a line that showed a significant difference with PS for each studied trait. When two NILs with overlapping introgressions showed significant effects, the QTL was located in the overlapping region. 


\section{Results}

About 300 root systems and 4500 images [six time points plus four different sections of each root: whole root, principal, secondary, and TRS (Fig. 1)] were studied in detail, representing on average of 10 plants per NIL and 20 of each parental line, PS and SC.

Phenotypic analysis of the parent genotypes. PS and SC showed strong differences in their growth patterns (Fig. 2). SC seeds were significantly smaller (reductions of $85 \%$ in SW) than PS seeds, giving rise to a proportionally reduced vine biomass ( $82 \%$ VW compared with PS; Figs. 2 and 3). Root size was also reduced in $\mathrm{SC}$, and despite the differences in plant size, the root-to-shoot ratio was similar in PS and in SC. In fact, we found proportional reductions in most of the whole root traits studied during the assay (Fig. 3), ranging from $75 \%$ to $82 \%$ in RL, from $70 \%$ to $90 \%$ in PA, and from $62 \%$ to $91 \%$ in TT, except for diameter (AD), which was reduced from $13 \%$ to $27 \%$. The development of the PR and of the SRS and TRS was altered (reductions at $8 \mathrm{~d}$ are represented in Fig. 4). However, not all traits showed size reductions proportional to those of the VW. The PR was affected in length (PRL reductions from 30\% to $68 \%$ ) and in diameter (PAD reductions from $33 \%$ to $45 \%$ ). Additionally, SC showed a delay in the development of the SRS. The diameter reduction was similar to that of PAD (SAD reductions from $30 \%$ to $36 \%$ ) and SRL was reduced proportionally to the reduction in plant size (from $80 \%$ to $81 \%$ ). However, the different traits of the SRS contributed differently to this reduction (from $34 \%$ to $80 \%$ for ALSR, from $57 \%$ to $66 \%$, for SRN, and from $54 \%$ to $71 \%$ for SRDe). A delay in tertiary root branching growth was also evident: from $68 \%$ to $100 \%$ in TRN, in SRT, and in $\%$ SRT, and from $66 \%$ to $90 \%$ in TRDe.

Heritabilities AND Correlations. For most traits, the heritability estimates were stable during the entire assay, except for diameter-related parameters, which showed important fluctuations with time (ranging from 0.18 to 0.56). WRS parameters showed moderate heritability estimates (0.29-
0.56), slightly higher than those obtained for traits studied in the primary, secondary, and tertiary root systems. The heritability estimates for PRL were low-to-moderate (0.19-0.37) and lower than those found for SRL (0.30-0.40) and ALSR (0.32$0.34)$. The SRN showed low-to-moderate heritability estimates (0.22-0.37), whereas the SRDe showed the lowest heritability estimates $(0.10-0.18)$. The heritabilities among the TRS traits were very similar (0.22-0.43). TRS and SRS heritabilities increased their value with time.

Phenotypic and genotypic correlations among root traits were calculated for each date and were stable during the entire assay (values at $8 \mathrm{~d}$ are shown in Table 1). SW was moderately correlated with many root traits, but phenotypic and genotypic correlations were higher for $\mathrm{VW}$ with parameters measuring length and branching of the WRS and of the SRS and TRS (except for some relative parameters such as SRDe and \%SRT). Traits of the WRS were highly phenotypically and genotypically correlated, except for AD. Significant but moderate genotypic correlations were found between parameters measuring length and diameter, in the primary (PRL and PAD) and SRS (SRL and ALSR, and SAD). The highest phenotypic and genotypic correlations among SRS traits occurred between the total SRL and SRN. It is interesting to note that the ALSR was only phenotypically and not genotypically correlated with SRL. SRDe only showed low-to-moderate phenotypic, and not genotypic, correlations with the other SRS parameters. In general, the traits of the TRS were highly phenotypically and genotypically correlated to each other (except for TRDe).

Regarding the correlations among parameters belonging to different categories, it is worth noting that traits measuring the size of the WRS (RL and PA) showed significant genotypic correlations with traits measuring length, diameter, and branching of the SRS and TRS (SRL, SAD, ALSR, TRN, and TRDe), and that TT showed moderate-to-high genotypic correlations with parameters that describe tertiary branching patterns. The PRL and PAD were highly genetically correlated with the extension of the SRS (SRN and SRL), but poorly correlated
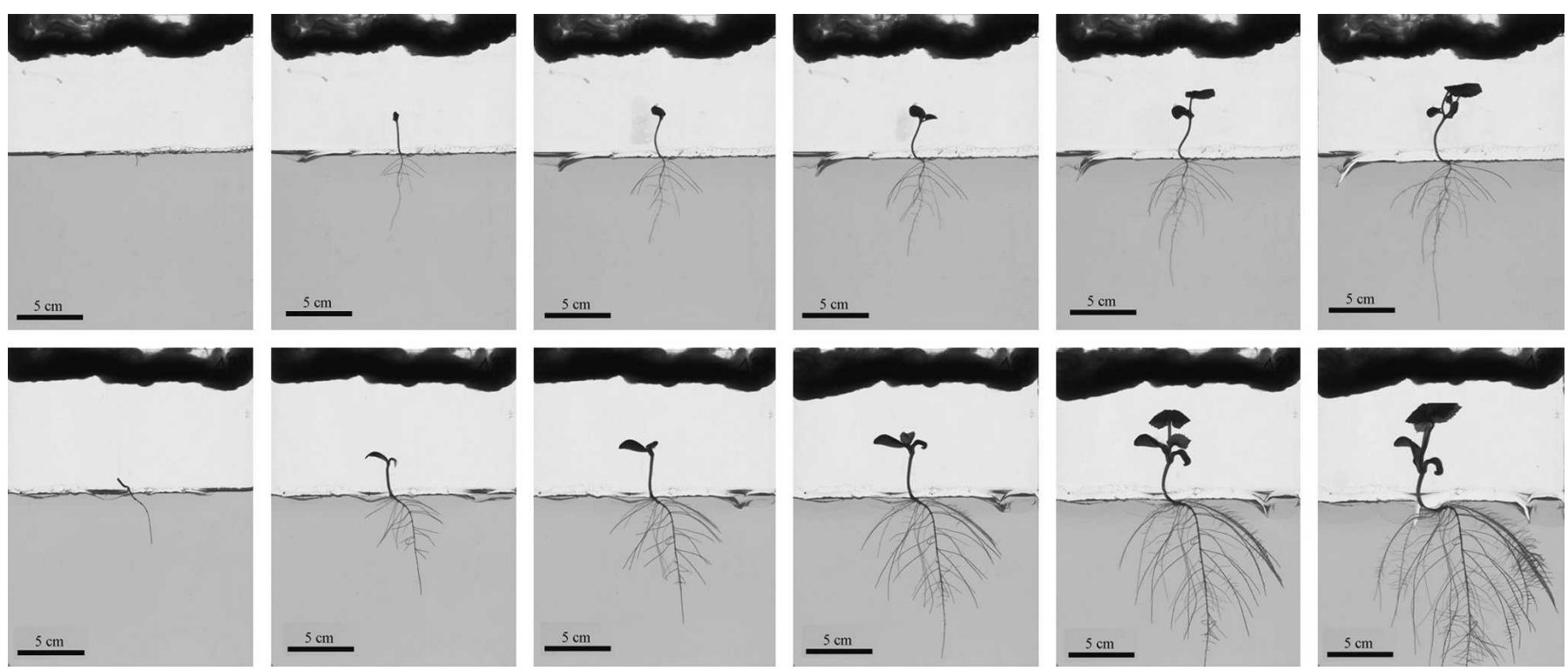
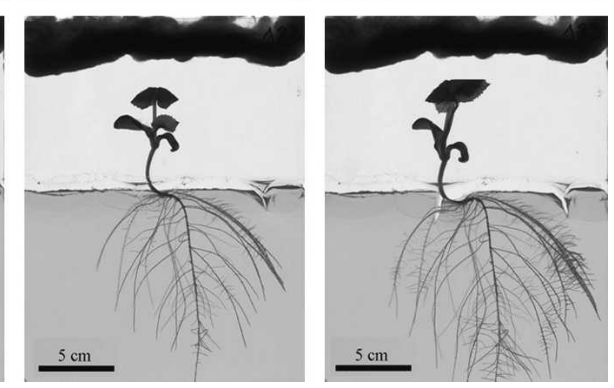

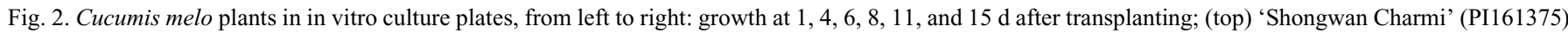
plants, (bottom) 'Piel de Sapo' plants. 


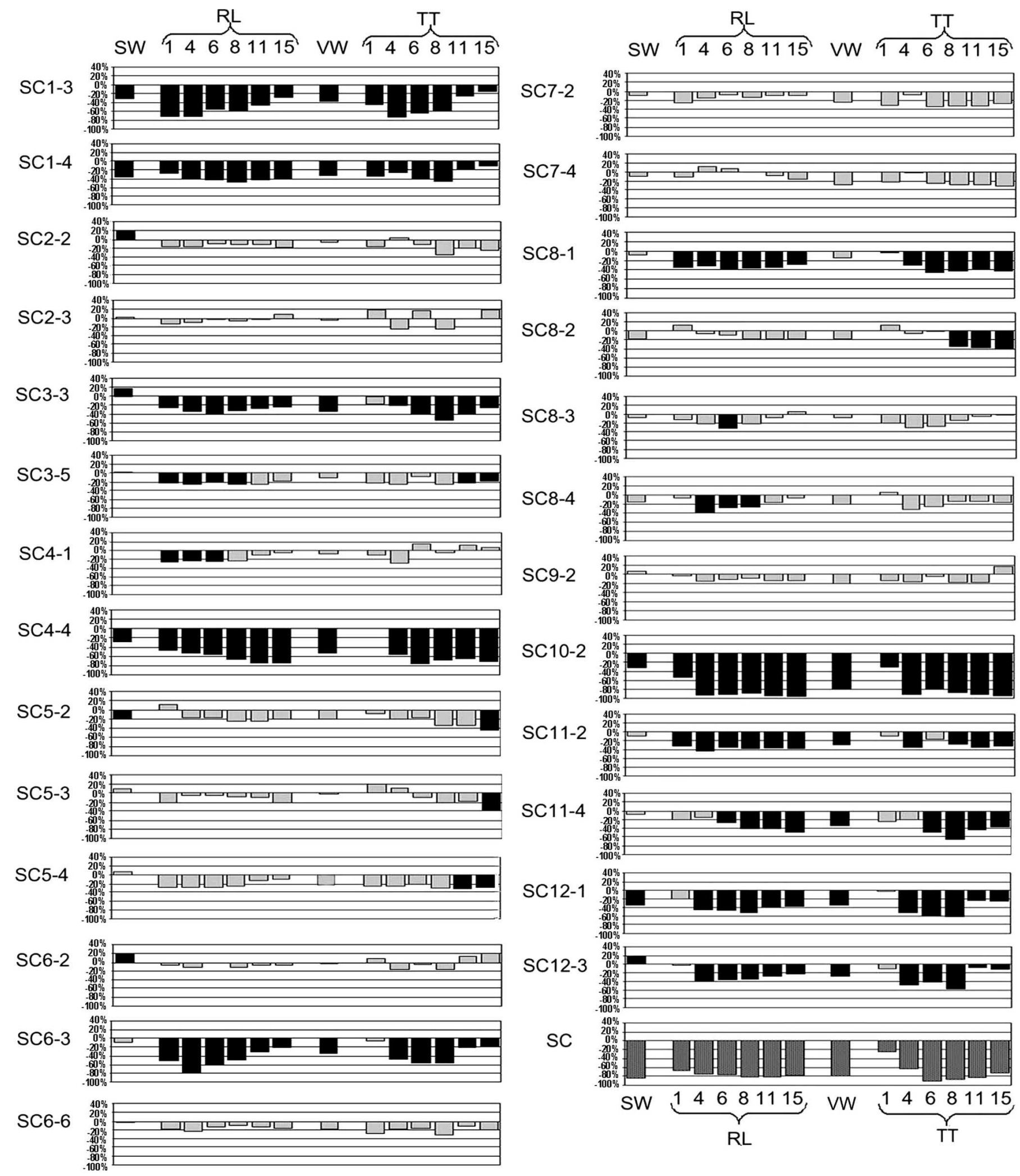

Fig. 3. Percentage of Cucumis melo 'Piel de Sapo' (PS) mean value for seed, vine, and root traits in C. melo 'Shongwan Charmi' [SC (PI161375)] and derived nearisogenic lines (NILs). Black and gray bars within NILs indicate whether differences from PS are significant or nonsignificant, respectively, by a Dunnet contrast with type-I error $\alpha \leq 0.05$ at 1, 4, 6, 8, 11, and $15 \mathrm{~d}$ after transplanting ( $\mathrm{SC}$ values are represented in dark gray bars, and all values were significantly different from those of PS). $\mathrm{SW}=$ seed weight, $\mathrm{RL}=$ total root length, $\mathrm{VW}=$ vine weight, $\mathrm{TT}=$ total number of root tips. The NILs had marked defined introgressions from SC into the PS background, which covered most of the SC genome (Eduardo et al., 2007) and were coded with the prefix "SC." The first number indicates the linkage group (LG), and a second one indicates the number of the NIL within the linkage group. 


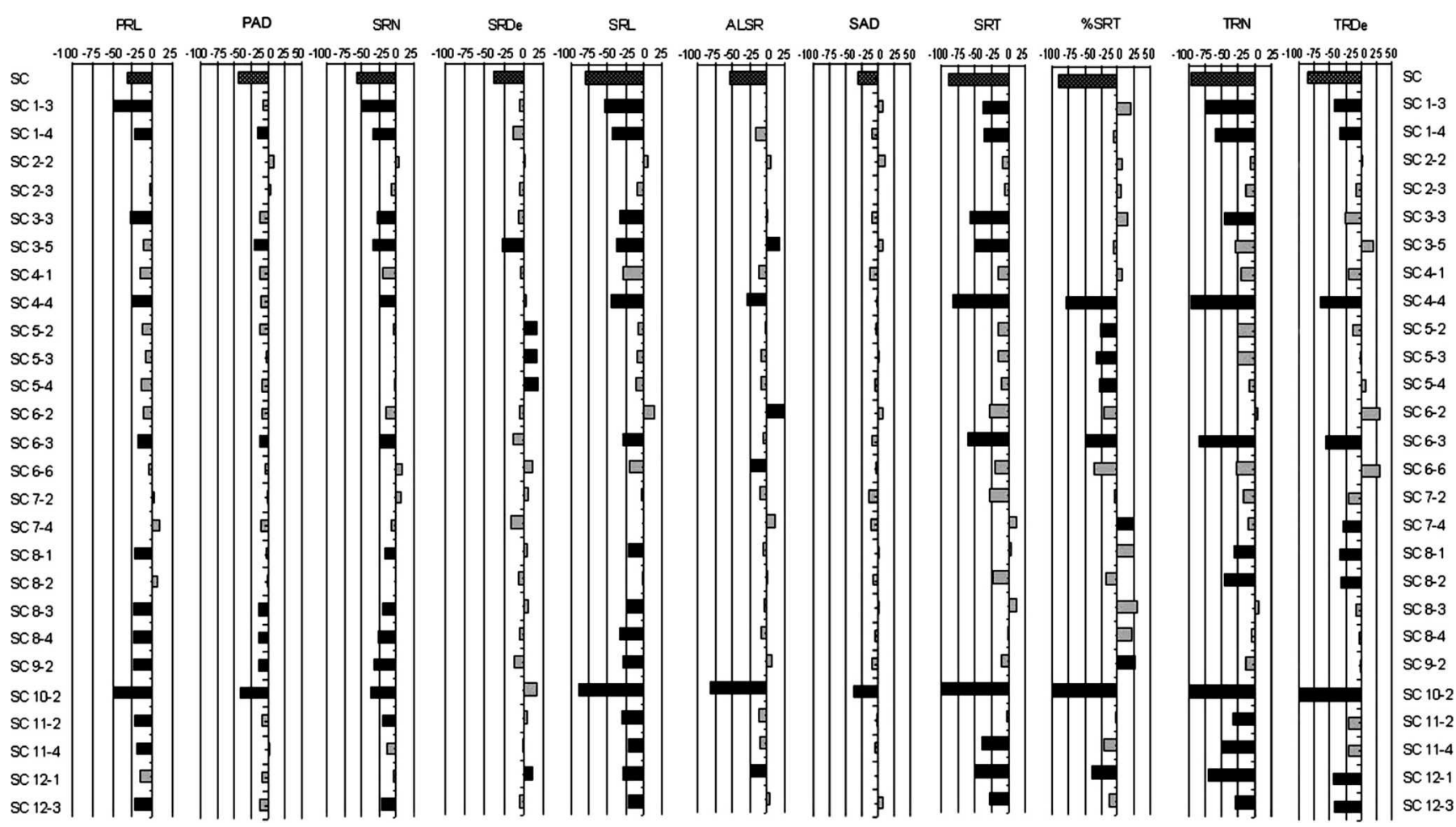

Fig. 4. Trait mean and statistical significance of Cucumis melo near-isogenic lines (NILs) compared with the control cultivar Piel de Sapo (PS) by a Dunnet contrast with type-I error $\alpha \leq 0.05$ at $8 \mathrm{~d}$ after transplanting. NIL names are shown on the left, traits are indicated above, and the percentage difference with respect to the control PS is shown above the graphics. Black and gray bars indicate whether differences are significant or nonsignificant, respectively [C. melo 'Shongwan Charmi' [SC (PI161375)] values are represented in dark gray bars, and all values were significantly different from those of PS]. $P R L=$ primary root length, PAD $=$ average diameter of primary root, $\mathrm{SRN}=$ number of secondary roots, $\mathrm{SRDe}=$ secondary root density calculated as $\mathrm{SRN}$ per PRL, SRL = total length of the secondary root system, ASRL = average length of secondary roots, $\mathrm{SAD}=$ average diameter of the lateral root system, $\mathrm{SRT}=$ number of secondary roots bearing tertiary roots, $\% \mathrm{SRT}=$ percentage of secondary roots bearing tertiary roots, $\mathrm{TRN}=$ number of tertiary roots, $\mathrm{TRDe}=$ tertiary root density calculated as $\mathrm{TRN}$ per SRT.

with SRDe. Also, ALSR and SAD were correlated with TRN and TRDe, but not with SRT or \%SRT.

Principal COMPONENT ANALYSIS AND ALlometric RELATIONSHIPS. PCA was useful for determining which combinations of attributes contributed more to the phenotypic diversity in the NILs population. The first three PCs contributed to a total of $65 \%$ of the observed variation. Traits with eigenvectors $>0.50$, considered the most significant contributors to each PC, are listed in Table 2. PC1, which represented $39 \%$ of the variation, was most affected by parameters describing vine biomass and size of the WRS, which is global plant size (VW, RL, PA, TT). Interestingly, parameters that reflect the degree of development of the TRS, except for the percentage of secondary roots bearing tertiaries, were also significant contributors to $\mathrm{PC} 1$ variation. Most of the parameters describing the growth of the principal root and the SRS contributed less (eigenvector $<0.5$ ) to $\mathrm{PC} 1$ variation. SW only partially influenced PC1, and SRDe did not significantly contribute to $\mathrm{PC} 1$ variation. Therefore, low values of PC1 correspond to NILs with reduced plant size, small root systems, and with a delayed development of the TRS. PC2 represented $16 \%$ of the variation and was affected positively by \%SRT and SAD and negatively by PRL and SRN (Table 2). High PC2 values correspond to root systems with a shorter PR, with fewer but thicker secondaries, and with a higher percentage of secondaries bearing tertiary roots. PC3, which represented $10 \%$ of the variation, was most affected by $\mathrm{AD}$ and $\mathrm{SAD}$.
According to the PCA results, variation in plant size (vine and root) explains a large proportion of the total variation found in the NIL population. Most of the NILs analyzed showed average VW, similar to PS. However, a set of NILs (SC1-3, SC1-4, SC3-3, SC4-4, SC6-3, SC10-2, SC11-2, SC11-4, SC121 , and $\mathrm{SC} 12-3$ ) showed a significant $\mathrm{VW}$ reduction, ranging from $33 \%$ to $85 \%$ of that of PS (Fig. 3). Previously, we described a high correlation between VW and most of the root traits, which reflects a significant effect of vine biomass variation on root values. In fact, the NILs with reduced VW displayed reductions in other root parameters (Figs. 3 and 4).

The allometric analysis allowed for a better characterization of the size-dependent variations between vine and roots in these two groups of NILs. Strong allometric relationships between VW and the group of traits measuring global size of the WRS (RL, PA, and TT) and of the secondary and TRS were found (SRL and TRN). However, NILs with a significant reduction of VW showed higher coefficients of determination (ranging from $r^{2}=48 \%$ to $68 \%$ ) in comparison with NILs without a significant reduction of VW ( $r^{2}=23 \%$ to $61 \%$ for the same traits). This suggests that these root traits maintain their proportionality to the vine biomass in both groups of lines. In general, allometric relationships were weaker for traits measuring diameter (AD, PAD, and SAD), PRL, SRN, ALSR, SRT, and TRDe. For these traits, the coefficients of determination were very low in the group of NILs without VW reduction $\left(r^{2}=3.4 \%\right.$ to $\left.15 \%\right)$ in comparison with the group of NILs that reduced VW $\left(r^{2}=11 \%\right.$ 


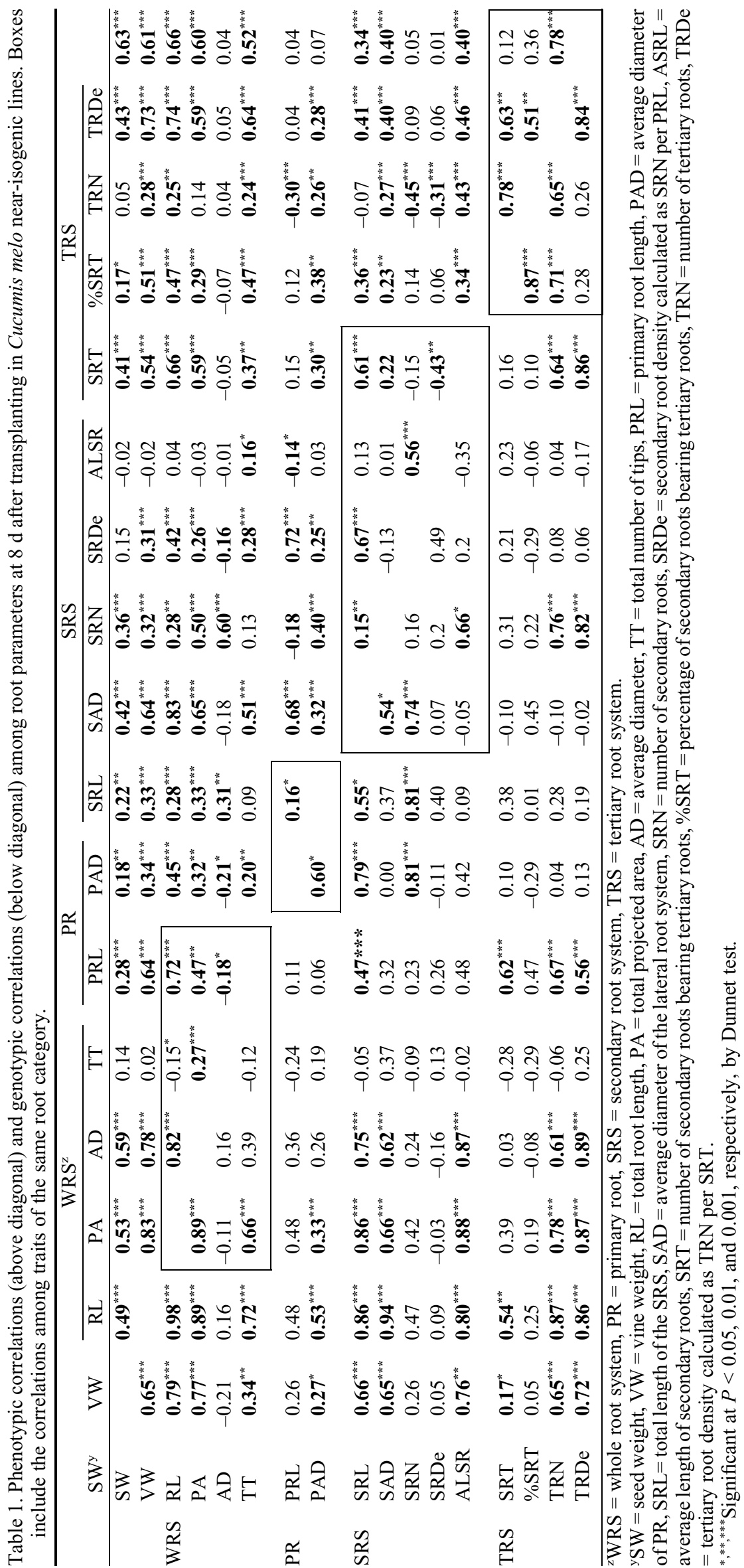


Table 2. Principal component analysis conducted on Cucumis melo near-isogenic lines. The percentage of the variability accounted for each principal component (PC1, PC2, and PC3) is listed with the acronym.

\begin{tabular}{|c|c|c|c|c|c|}
\hline \multicolumn{2}{|c|}{ PC1 $(39 \%)$} & \multicolumn{2}{|c|}{ PC2 (16\%) } & \multicolumn{2}{|c|}{ PC3 (10\%) } \\
\hline Trait $^{z}$ & $\overline{\text { Eigenvector }}$ & Trait & $\overline{\text { Eigenvector }}$ & Trait & $\overline{\text { Eigenvector }}$ \\
\hline$\overline{\mathrm{RL}}$ & 0.96 & $\%$ SRT & 0.71 & $\mathrm{AD}$ & 0.71 \\
\hline VW & 0.87 & SAD & 0.55 & SAD & 0.61 \\
\hline $\mathrm{PA}$ & 0.85 & PRL & -0.65 & & \\
\hline TRN & 0.84 & SRN & -0.82 & & \\
\hline SRL & 0.79 & & & & \\
\hline TT & 0.73 & & & & \\
\hline TRDe & 0.69 & & & & \\
\hline SRT & 0.64 & & & & \\
\hline ALSL & 0.61 & & & & \\
\hline SW & 0.54 & & & & \\
\hline
\end{tabular}

${ }^{\mathrm{z}}$ Traits that contributed most to each principal component (eigenvector $>0.5)$; $\mathrm{RL}=$ total root length, $\mathrm{VW}=$ vine weight, $\mathrm{PA}=$ total projected area, $\mathrm{TRN}=$ number of tertiary roots, $\mathrm{SRL}=$ total length of the secondary root system, TT $=$ total number of tips, $\mathrm{TRDe}=$ tertiary root density calculated as TRN per SRT, SRT $=$ number of secondary roots bearing tertiary roots, ASRL = average length of secondary roots, SW $=$ seed weight, $\% \mathrm{SRT}=$ percentage of secondary roots bearing tertiary roots, $\mathrm{SAD}=$ average diameter of the lateral root system, $\mathrm{PRL}=$ primary root length, $\mathrm{SRN}=$ number of secondary roots, $\mathrm{AD}=$ average diameter, $\mathrm{PAD}=$ average diameter of the primary root, $\mathrm{SRDe}=$ secondary root density calculated as SRN per PRL.

to $40 \%$ ). The only trait for which the allometric coefficient was not significant in both groups of NILs was SRDe. We also studied the allometric relationships of root traits with SW, but did not find significant allometric coefficients (data not shown).

Allometric relationships and principal component analysis results indicate that NILs with a reduction in VW exhibit proportional reductions in most of the root traits, and that this variation in plant size accounts for most of the variation observed in the population of NILs. This scaling effect masks the size-independent variation in root structure due to genetic differences of these lines with PS. In contrast, lines with VW similar to PS showed weaker allometric relationships between VW and most of the traits (except for some absolute traits measuring global root size). All of these lines grouped with PS in the principal component analysis according to the $\mathrm{PC} 1$ axis.

To localize in the melon genome the QTLs responsible for the root variation not masked by differences in size, in the next sections, we analyze in detail the variation observed in the NIL population taking into consideration the allometric and PCA information. As a general criterion, NILs with a general reduction of plant size are not considered for the QTL analysis of root traits. However, we can consider that these lines carry QTLs affecting VW, in a minimum number of seven. Additionally, in the remaining lines, we analyze those root traits for which weak allometric relationships with VW suggest the importance of genotypic variation.

\section{QTL analysis}

Primary Root Length. As expected, almost all the lines with reduced plant size showed significant reductions in PRL in comparison with PS, except for SC12-1 (Fig. 4). Among the NILs with a plant size similar to PS, four (SC8-1, SC8-3, SC84, and SC9-2) showed a significant reduction in PRL $(20 \%-$ $25 \%$ ). SC8-3 and SC8-4 share a fragment of the introgression, so they may represent a single QTL. The estimated minimum number of QTLs affecting PRL in these lines is three (Figs. 5 and 6).

Primary root diameter. Among the lines that showed a similar size to PS, only SC3-5, SC8-3, SC8-4, and SC9-2 showed a significant reduction of PAD (Fig. 4), defining a minimum number of QTLs of three (Fig. 5).

Number AND DENSITY OF SECONDARY ROOTS. Many NILs showed a significant reduction in SRN. This reduction was, in general, the consequence of a reduced PRL. In fact, these two traits were highly correlated, whereas SRDe only varied in five of these lines (Fig. 4). A significant reduction of SRDe was observed in SC3-5. In contrast, a transgressive inheritance for SRDe was found in NILs in LG 5 (SC5-2, SC 5-3, and SC5-4) and in SC12-1, where increases of up to $20 \%$ over PS were observed. Therefore, the minimum number of QTLs affecting root density is three, as SC5-2, SC5-3, and SC5-4 share a fragment, two with a positive effect and one with a negative effect (Figs. 5 and 6). The QTLs for SRDe in SC12-1, a line with VW reduction, was considered to be due to the lack of significant allometric relationships between VW and SRDe and the lack of significant reduction in PRL.

LENGTH OF THE SECONDARY ROOT SYSTEM. Most of the NILs with reduced PRL and SRN showed a proportional reduction of the SRL. QTLs were not considered for SRL as the three traits are highly correlated and allometric relationships were significant for this trait and VW. A group of NILs without a reduction of VW showed a significant variation in ALSR: SC3-5, SC6-2, and SC6-6. NILs of LG 6 significantly increased (SC6-2) and decreased (SC6-6) ALSR without significantly altering SRN or SRDe, whereas SC3-5 had an increase in ALSR associated to a decrease in SRDe. Therefore, we can estimate that two QTLs that increase in ALSR exist in SC6-2 and SC3-5, and one QTL that reduces the length exists in SC6-6 (Figs. 5 and 6).
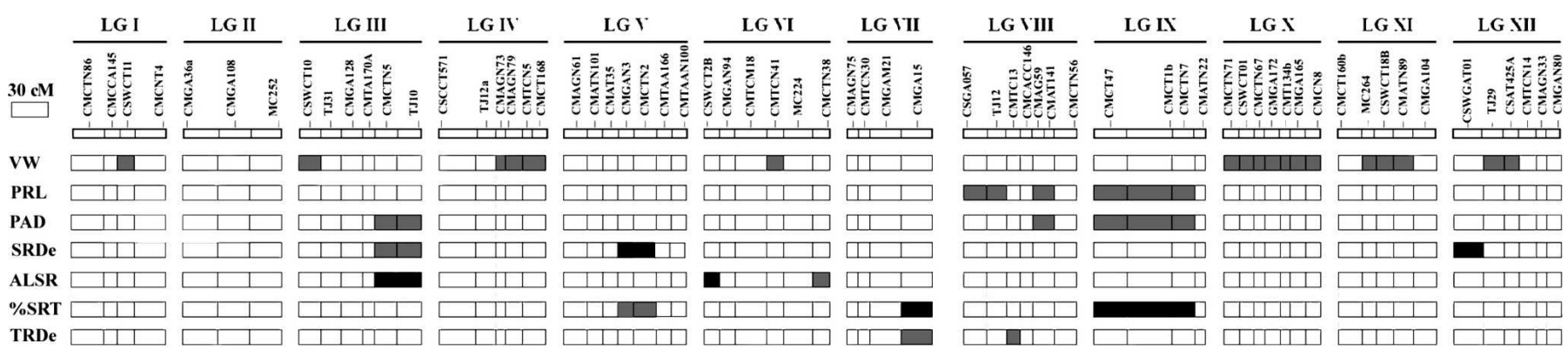

Fig. 5. Ideogram showing the location of vine weight and root trait loci, located by means of near-isogenic lines on the Cucumis melo genetic map. Linkage groups (LG) are depicted at the top according to Gonzalo et al., (2005). For each trait, the estimated position of the QTL is indicated by a shadowed area and is colored black when the 'Shongwan Charmi' (SC) allele increased the trait and gray when it decreased the trait with respect to 'Piel de Sapo' (PS). 

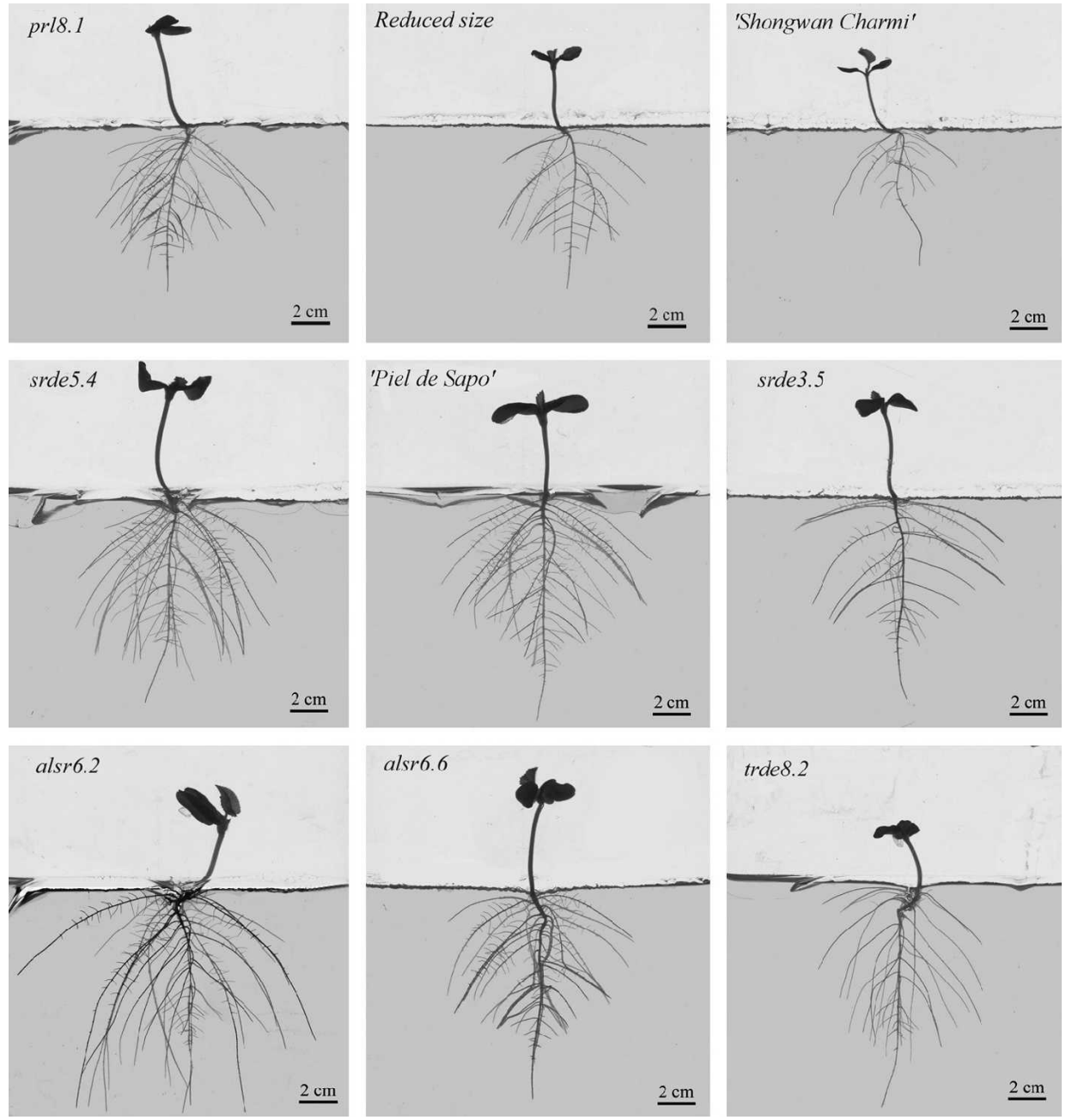

Fig. 6. Plants of different near-isogenic lines, Cucumis melo 'Shongwan Charmi' (PI161375; top right corner) and C. melo 'Piel de Sapo' (center) showing the effects of different QTLs over the background of PS ( $8 \mathrm{~d}$, in vitro culture). Names in italics correspond to QTLs found, the first number indicates the linkage group (LG), and a second one indicates the number of NIL within the LG. prl8.1=QTL for reduced principal root length, reduced size $=$ NIL that showed a general reduced size in comparison with 'Piel de Sapo', srde5.4=QTL for enhanced secondary root density, srde3.5 = QTL reduced secondary root density, alsr6.2 = QTL enhanced average length of the secondary roots, alsr6.6 = QTL of reduced average length of the secondary roots, trde8.2 $=$ QTL of reduced tertiary root density.

Diameter OF THE SECONDARY ROOTS. SAD was not very variable. Slight increases or decreases, associated with the variation of ALSR, were observed. A significant reduction in SAD was only observed in SC10-2, which developed secondary roots thinner than PS, and very similar to SC (Fig. 4).

Tertiary RoOT SYSTEM. All of the NILs with a general reduction in plant size showed a delay in the development of the TRS (Fig. 4). In the NILs with plant size similar to PS, QTLs were considered for \%SRT and TRDe, which showed weak allometric relationships with VW. NILs of LG 5 significantly reduced the \%SRT, associated with an increase in SRDe. The opposite effect was observed in SC9-2 and SC7-4. In the latter (SC7-4), this effect was also associated with a reduction in TRDe. Finally, lines SC8-1 and SC8-2 showed a reduction in TRDe without altering the \%SRT. NILs of LG 5 share a fragment of the introgression, and so the minimum number of QTLs affecting the \%SRT is three, one with a negative effect and two with a positive effect. Also, as SC8-1 and SC8-2 share a fragment, the minimum number of QTLs affecting TRDe is two, both with negative effects (Figs. 4-6).

\section{Discussion}

Previous studies of root architecture in melon were carried out using destructive assays by unearthing root systems from adult plants of $\mathrm{F}_{2}$ or $\mathrm{F}_{2: 3}$ families grown in the field or in pots (Dias et al., 2004; Fita et al., 2006). This is the first study in which NILs and in vitro culture have been used for the study of root genetics in melon. In vitro culture offers many advantages for investigating roots: a precise control of environmental variation, a nondestructive, extremely easy visualization, and an accurate evaluation of the dynamics of the root system. The main shortcoming is that the growth media are unnatural. However, the root structures found for the parental lines when analyzing plants grown in fields and in greenhouses (Dias et al., 2002, 2004; Fita et al., 2006; Iglesias et al., 2000) were similar to those described here. Also, many authors have noticed that, in most cases, the genetic rankings, if not the absolute values for root parameters, are robust across environments (Rubio et al., 2003; Tuberosa et al., 2003; Zhu et al., 2005).

The heritability estimates provide an overview of the genetic control of the traits under study. In general, root parameters showed moderate heritabilities, indicating that it is possible to perform a successful selection for these traits. Fluctuations observed in the heritability estimates of diameter parameters can be explained by considering the effect of the branching process on the diameter and the errors in the measurements, a consequence of the limitations of the image analysis (Bouma et al., 2000).

Interestingly, the heritabilities for parameters of the SRS and TRS increased over days. This is probably a consequence of small temporal lags in the branching process between plants of the same NIL during the early phases of root branching. Eight days after transplanting, we reach the highest heritability values. At later stages, image analysis of the whole root is open to more errors due to the greater complexity of the root systems. The evolution of heritability throughout the development may also be due to the different heritability of the genes expressed in the different developmental stages.

The heritabilities for RL, PA, and SRN are similar to those obtained in adult melon plants using $\mathrm{F}_{2: 3}$ populations (Dias et al., 2004; Fita et al., 2006). We do not have previous information about the heritability of the remaining traits in melon. Heritabilities for the length of the PR and the SRS and 
the number of secondaries were lower than those found by other authors in large RIL populations of $A$. thaliana, maize, and rice (Fitz-Gerald et al., 2006; Loudet et al., 2005; Price et al., 1997; Tuberosa et al., 2002). This is the first time, to our knowledge, that the heritability of tertiary parameters has been estimated.

Phenotypic and genotypic correlations also provide valuable information from a genetic and breeding standpoint. Our results agree with those obtained in other species. The appearance of laterals seems to depend on the diameter and the length of the bearing root, as indicated by the correlations between the length and diameter of the primary and secondary roots with the number of secondary and tertiary roots, respectively. These results, along with the lack of genetic correlation between the PRL and the SRDe, agree with those of Loudet et al. (2005), who found an association between the PRL and the number of laterals, but not with the density of laterals. Correlation analysis also indicates that most of the variation observed in our population in the SRL comes from variation in the SRN, and less from variation in the ALSR or SRDe. It is interesting to note the lack of correlation between the SRN and SRDe and between the SRT and the TRDe.

PCA analysis showed that a large part of the NIL variation was due to the differences in plant size, including $\mathrm{VW}$ and whole root traits. The NILs SC1-3, SC1-4, SC3-3, SC4-4, SC63, SC10-2, SC11-2, SC11-4, SC12-1, and SC12-3 showed important reductions in size or a developmental delay in comparison with PS. The results of the allometric study reinforced the idea that plant size variation can mask sizeindependent variation of root traits. Scaled and relative parameters have been successfully used in other species in the comparison of roots of different sizes (Johnson et al., 2000). In our study, most root parameters showed strong allometric relationships with VW in the group of NILs that significantly reduced VW, except for the SRDe. Fortunately, allometric relationships were weaker for most traits in the group of NILs with size similar to PS. The analysis of these NILs has provided QTLs affecting root traits without the confounding effects of the differences in plant size. Thus, we have found six QTLs affecting the PR in LGs 3, 8, and 9. In all cases, the effect of the $\mathrm{SC}$ introgression was negative. The high genotypic correlation between the length and the diameter of the PR, along with the colocalization of prl9.2 with pad9.2, and prl8.3.4 with pad8.3.4, may suggest that these traits could be controlled by the same gene(s). Nevertheless, the independence of prl8.1 and pad3.5 indicates that it is possible to change the PRL and PAD independently.

Regarding the SRS, we found three QTLs for the density of secondaries (LGs 3, 5, and 12) and three QTLs for the average length of secondaries (LGs 3 and 6). In both cases, there are QTLs with a positive effect (two for SRDe and two for ALSR) masked in the SC genetic background. Transgressive values are commonly found in QTL analysis, and demonstrate the utility of introgression lines in taking advantage of hidden variation in genetic resources (Eduardo et al., 2007; Fitz-Gerald et al., 2006). Loudet et al. (2005) also found transgressive values for SRDe. Moreover, it is interesting to point out the colocalization of srde3.5 with alsr3.5. The fact that SRDe and ALSR change in opposite directions seems to indicate a pleiotropic effect. However, as occurred in the PR traits, the existence of additional independent QTLs for SRDe (srde5.3.4) and ALSR (alsr6.2 and alsr6.6), in addition to the lack of a significant genotypic correlation between these two traits, indicates that it is possible to change the length of the laterals and their density independently.

Regarding the TRS, we found two QTLs for the density of tertiaries (trde7.4 and trde8.1.2) and three QTLs for the percentage of secondaries with tertiaries, one negative (\%srt5.3.4) and two positive (\%srt7.4 and \%srt9.2). It is interesting to note the transgressive effect on \%SRT in lines SC7-4 and line SC9-2.

Many colocalizations appear in different lines among parameters of different root categories (in LGs 3, 5, and 9): pad3.5 with srde3.5 and alsr3.5 and srde5.3.4 with \%srt5.3.4 and prl9.2 with pad9.2 and \%srt9.2. However, the colocalization always has to be interpreted with care because it could be pleiotropy or linkage. To dissect those effects, it is necessary to study subNILs. Fortunately, the absence of colocalization is very informative. This is the case of the clear independence of QTLs controlling SRDe and PRL. Loudet et al. (2005) had the same results finding colocalization of QTLs in A. thaliana for the PRL with the SRN, but not with the SRDe.

In summary, we describe a total of 17 QTLs affecting root traits. This study provides a starting point for further characterization of the genetic factors involved in root architecture in melon. In other crops, QTLs for root traits, such as root length, enhanced lateral rooting, and adventitious rooting, have shown to be useful for breeding against soil stresses such as phosphorous or water deficiency (Beebe et al., 2006; Zhu and Lynch, 2004). For the first time, a newly available melon NIL library has been used to study root traits. Future studies with subNILs derived from the NILs of greatest interest selected in this study and in different growing conditions will allow the use of allelic variability present in melon germplasm for modifying the root architecture of cultivated melons as a way to breed melons with more efficient root systems.

\section{Literature Cited}

Argyris, J., M.J. Trucol, O. Ochoa, S.J. Knapp, D.W. Still, G.M. Lenssen, J.W. Schut, R.W. Michelmore, and K.J. Bradford. 2005. Quantitative trait loci associated with seed and seedling traits in Lactuca. Theor. Appl. Genet. 111:1365-1376.

Beebe, S.E., M. Rojas-Pierce, X. Yan, M.W. Blair, F. Pedraza, F. Muñoz, J. Tohme, and J. Lynch. 2006. Quantitative trait loci for root architecture traits correlated with phosphorus acquisition in common bean. Crop Sci. 46:413-423.

Bouma, T.J., K.L. Nielsen, and B. Koutstaal. 2000. Sample preparation and scanning protocol for computerised analysis of root length and diameter. Plant Soil 218:185-196.

Clarke, J.M. and T.N. McCaig. 1993. Breeding for efficient root systems, p. 485-499. In: M.D. Hayward, N.O. Bosemark, and I. Romagosa (eds.). Plant breeding: Principles and prospects. Chapman and Hall, London.

Dias, R., C.S. De, B. Picó, J. Herraiz, A. Espinós, and F. Nuez. 2002. Modifying root structure of cultivated muskmelon to improve vine decline resistance. HortScience 37:1092-1097.

Dias, R.C.S., B. Picó, A. Espinós, and F. Nuez. 2004. Resistance to melon vine decline derived from Cucumis melo ssp. agrestis: Genotypic analysis for root structure and root response. Plant Breed. 123:66-72.

Dunnet, C.W. 1955. A multiple comparison procedure for comparing several treatments with a control. J. Amer. Stat. Assn. 50:1096-1121.

Eduardo, I., P. Arus, and A.J. Monforte. 2005. Development of a genomic library of near-isogenic lines (NILs) in melon (Cucumis melo L.) from the exotic accession SC. Theor. Appl. Genet. 6:1-10. Eduardo, I., P. Arús, A.J. Monforte, J. Obando, J.P. FernándezTrujillo, J.A. Martínez, A.L. Alarcón, J.M. Álvarez, and E. Van 
der Knaap. 2007. Estimating the genetic architecture of fruit quality traits in melon using a genomic library of near-isogenic lines. J. Amer. Soc. Hort. Sci. 132:80-89.

Eshed, Y. and D. Zamir. 1995. An introgression line population of Lycopersicon pennellii in the cultivated tomato enables the identification and fine mapping. Genetics 141:1147-1162.

Fernández-Trujillo, J.P., J. Obando, J.A. Martínez, A.L. Alarcón, I. Eduardo, P. Arús, and A.J. Monforte. 2007. Mapping fruit susceptibility to postharvest physiological disorders and decay using a collection of near-isogenic lines of melon. J. Amer. Soc. Hort. Sci. 132:1-10.

Fita, A., B. Pico, and F. Nuez. 2006. Implications of the genetics of root structure in melon breeding. J. Amer. Soc. Hort. Sci. 131:372379.

Fita, A., B. Picó, and F. Nuez. 2007. Melon roots under stress: Melon vine decline. Plant Stress 1:93-104.

Fitter, A. 2002. Characteristics and functions of root systems, p. 15-32. In: Y. Waisel, A. Eshel, and U. Kafkafi (eds.). Plant roots: The hidden half. Marcel and Decker, New York.

Fitz-Gerald, J.N., M.D. Lehti-Shiu, P.A. Ingram, K.I. Deak, T. Biesiada, and J.E. Malamy. 2006. Quantitative trait loci that regulate Arabidopsis root system size and plasticity. Genetics 172:485-498.

Gómez-Guillamón, M.L. and J.M. Álvarez. 2001. Melón, p. 194 198. In: F. Nuez and G. Llácer (eds.). La horticultura española. Ediciones de horticultura. Barcelona, Spain.

Gonzalo, M.J., M. Oliver, J. García-Mas, A. Monfort, R. DolcetSanjuan, N. Katzir, P. Arus, and A.J. Monforte. 2005. Simplesequence repeat markers used in merging linkage maps of melon (Cucumis melo L.). Theor. Appl. Genet. 110:802-811.

Harper, J.L., M. Jones, and N.R. Hamilton. 1991. The evolution of roots and the problem of analysing their behaviour, p. 3-24. In: D. Atkinson (ed.). Plant root growth: An ecological perspective. British Ecological Society Spec. Publ. No.10. Blackwell, Oxford, UK.

Hébert, D., S. Fauré, and I. Olivieri. 1994. Genetic, phenotypic and environmental correlations in black medic, Medicago lupulina L., grown in three different environments. Theor. Appl. Genet. 88:604613.

Hunt, R. 1990. Basic growth analysis: Plant growth analysis for beginners. 1st ed. Unwin Hyman, London.

Iglesias, A., B. Picó, and F. Nuez. 2000. A temporal genetic analysis of disease resistance genes: Resistance to melon vine decline derived from Cucumis melo var agrestis. Plant Breed. 118:1-6.

Jeffrey, C. 1980. A review of the Cucurbitaceae. Bot. J. Linnean Soc. 81:233-247.

Johnson, W.C., L.E. Jackson, O. Ochoa, R. Van Wijk, J. Peleman, D.A. St.Clair, and R.W. Michelmore. 2000. Lettuce, a shallow-rooted crop, and Lactuca serriola, its wild progenitor, differ at QTL determining root architecture and deep soil water exploitation. Theor. Appl. Genet. 101:1066-1073.

Kempthorne, O. 1969. An introduction to genetic statistics. Iowa State University Press. Ames, IA.

Landi, P., M.C. Sanguineti, C. Liu, Y. Li, T.Y. Wang, S. Giuliani, M. Bellotti, S. Salvi, and R. Tuberosa. 2007. Root-ABA1 QTL affects root lodging, grain yield, and other agronomic traits in maize grown under well-watered and water-stressed conditions. J. Expt. Bot. 58:319-326.

Langlade, N.B., X. Feng, T. Dransfield, L. Copsey, A.I. Hanna, C. Thébaud, A. Bangham, A. Hudson, and E. Coen. 2005. Evolution through genetically controlled allometry space. Proc. Natl. Acad. Sci. USA 102:10221-10226.

Li, Z., P. Mu, C. Li, H. Zhang, Z. Li, Y. Gao, and X. Wang. 2005. QTL mapping of root traits in a doubled haploid population from a cross between upland and lowland japonica rice in three environments. Theor. Appl. Genet. 110:1244-1252.
Loudet, O., V. Gaudon, A. Trubuil, and F. Daniel-Vedele. 2005. Quantitative trait loci controlling root growth and architecture in Arabidopsis thaliana confirmed by heterogeneous inbred family. Theor. Appl. Genet. 110:742-753.

Lynch, J. 1995. Root architecture and plant productivity. Plant Physiol. 109:7-13.

Malamy, J. 2005. Intrinsic and environmental response pathways that regulate root system architecture. Plant Cell Environ. 28:67-77.

Obando, J., J.P. Fernández-Trujillo, J.A. Martinez, A.L. Alarcón, I. Eduardo, P. Arús, and A.J. Monforte. 2008. Identification of melon fruit quality quantitative trait loci using near-isogenic lines. J. Amer. Soc. Hort. Sci. 133:139-151.

Ochoa, I.E., M.W. Blair, and J.P. Lynch. 2006. QTL analysis of adventitious root formation in common bean under contrasting phosphorus availability. Crop Sci. 46:1609-1621.

Picó, B., A. Fita, and F. Nuez. 2007. Using genetics to improve stress resistance through altering root architecture. CAB Reviews: Perspectives Agr. Veterinary Sci. Nutr. Natural Resources 2(053). 15 Feb. 2008. <http://www.cababstractsplus.org/CABReviews/ Reviews.asp? action=display\&openMenu=relatedItems\&ReviewID= $32700 \&$ Year $=2007 \#>$.

Price, A.H., A.D. Tomos, and D.S. Virk. 1997. Genetic dissection of root growth in rice (Oryza sativa L.). I. A hydroponic screen. Theor. Appl. Genet. 95:132-142.

Rubio, G., H. Liao, X. Yan, and J. Lynch. 2003. Topsoil foraging and its role in plant competitiveness for phosphorous in common bean. Crop Sci. 43:598-607.

Steele, K.A., A.H. Price, H.E. Shashidhar, and J.R. Witcombe. 2006. Marker-assisted selection to introgress rice QTLs controlling root traits into an Indian upland rice variety. Theor. Appl. Genet. 112:208-221.

Tanksley, S.D. and J.C. Nelson. 1996. Advanced backcross QTL analysis: A method for the simultaneous discovery and transfer of valuable QTLs from unadapted germplasm into elite breeding lines. Theor. Appl. Genet. 92:191-203.

Tuberosa, R., S. Salvi, M.C. Sanguineti, M. Maccaferri, M. Giuliani, and P. Landi. 2003. Searching for quantitative trait loci controlling root traits in maize: A critical appraisal. Plant Soil 255:35-54.

Tuberosa, R., M.C. Sanguineti, P. Landi, M.M. Giuliani, S. Salvi, and S. Conti. 2002. Identification of QTLs for root characteristics in maize grown in hydroponics and analysis of their overlap with QTLs for grain yield in the field at two water regimes. Plant Mol. Biol. 48:697-712.

Zheng, B.S., L. Yang, W.P. Zhang, C.Z. Mao, Y.R. Wu, K.K. Yi, F.Y. Liu, and P. Wu. 2003. Mapping QTLs and candidate genes for rice root traits under different water-supply conditions and comparative analysis across three populations. Theor. Appl. Genet. 107:15051515 .

Zhu, J. and J.P. Lynch. 2004. The contribution of lateral rooting to phosphorous acquisition efficiency in maize (Zea mays) seedlings. Funct. Plant Biol. 31:949-958.

Zhu, J., S.M. Kaeppler, and J.P. Lynch. 2005. Mapping QTLs for lateral root branching and length in maize (Zea mays L.) under differential phosphorous supply. Theor. Appl. Genet. 111:688-695.

Zobel, R.W. 2005a. Primary and secondary root systems, p. 3-14. In: S.F. Wright and R.W. Zobel (eds.). Roots and soil management: Interactions between roots and the soil. Agron. Monograph 48. Amer. Soc. Agron., Crop Sci. Soc. Amer., and Soil Sci. Soc. Amer., Madison, WI.

Zobel, R.W. 2005b. Tertiary root systems, p. 35-56. In: S.F. Wright and R.W. Zobel (eds.). Roots and soil management: Interactions between roots and the soil. Agron. Monograph 48. Amer. Soc. Agron., Crop Sci. Soc. Amer., and Soil Sci. Soc. Amer., Madison, WI. 